\title{
Epidemic Spreading Characteristics and Immunity Measures Based on Complex Network with Contact Strength and Community Structure
}

\author{
Xueting Zhang, ${ }^{1}$ Bingfeng Ge, ${ }^{1}$ Qi Wang, ${ }^{2}$ Jiang Jiang, ${ }^{1}$ Hanlin You, ${ }^{1}$ and Yingwu Chen ${ }^{1}$ \\ ${ }^{1}$ College of Information System and Management, National University of Defense Technology, Changsha, Hunan 410073, China \\ ${ }^{2}$ College of Electronic Science and Engineering, National University of Defense Technology, Changsha, Hunan 410073, China \\ Correspondence should be addressed to Xueting Zhang; zhangxueting0807@foxmail.com
}

Received 3 July 2015; Accepted 22 October 2015

Academic Editor: Xiaobo Qu

Copyright (C) 2015 Xueting Zhang et al. This is an open access article distributed under the Creative Commons Attribution License, which permits unrestricted use, distribution, and reproduction in any medium, provided the original work is properly cited.

Middle East Respiratory Syndrome (MERS), bursting in South Korea from May 2015 and mainly spreading within the hospitals at the beginning, has caused a large scale of public panic. Aiming at this kind of epidemic spreading swiftly by intimate contact within community structure, we first established a spreading model based on contact strength and SI model, and a weighted network with community structure based on BBV network model. Meanwhile, the sufficient conditions were deduced to ensure the optimal community division. Next, after the verification by the real data of MERS, it is found that the spreading rate is closely related to the average weight of network but not the number of communities. Then, as the further study shows, the final infection proportion declines with the decreases both in isolation delay and in average weight; however, this proportion can only be postponed rather than decreased with respect to sole average weight reduction without isolation. Finally, the opportunities to take action can be found to restrain the epidemic spreading to the most extent.

\section{Introduction}

Middle East Respiratory Syndrome (MERS), first identified in Saudi Arabia in 2012, is a viral respiratory disease caused by a novel coronavirus. According to the World Health Organization (WHO), on May 14, 2013, there were 38 MERS cases, which grew further to 1150 after two years (i.e., May 31, 2015). For human-to-human transmission, the virus does not appear to pass easily from person to person unless they have close contact, such as providing unprotected care to or living together with infected patients.

The epidemic status in South Korea from May 20, 2015, caused national public panic and worldwide attention. At the beginning, it spread evidently amongst the infectors and the patients in the same sickroom. This paper thus focuses on the spreading characteristics of intimate contact with community structure.

Nowadays, the studies of epidemics spreading are twofold: the spreading model of differential equation and the complex network theory. There are three spreading models widely used in the study of virus transmission, namely, SIR model, SIS model, and SI model [1-4], and the solving algorithms are mainly based on percolation theory $[5,6]$, mean field theory $[7,8]$, and Markov chain theory $[9,10]$. For the complex network, Erdös [11] proposed the random network, while Watts and Strogatz [12] presented the small world network model with smaller average shortest path length and bigger clustering coefficient. Barabási and Albert [13] put forward the scale-free network model with both adding points and preferential attachment. According to the real network, the connections in many networks are not merely binary entities (i.e., either present or not) but have associated weights that record their strengths relative to one another. Thus, Barrat et al. [14] created the BBV model where point weight and edge weight evolve dramatically.

With the further study on the network topology, it is widely recognized that closely connected nodes and communities in social networks play an important role in topological properties and functional dynamics of involved complex networks $[15,16]$. As a result, there are many community 
division algorithms and accuracy indices such as modularity [17-20]. Based on the aforementioned models, Liu and $\mathrm{Hu}$ [21] researched on the epidemic spreading through the network with small world effect by SIS model, while Smieszek et al. [22] studied that by SIR model. Salathé and Jones [23] focused on the influence of community structure to virus transmission. In order to know about the virus transmission mechanism better, the dynamic models endeavor to slow down the outbreak rate and control the spreading range. Many different immunization strategies are proposed, such as random immunization [1], target immunization [8], and acquaintance immunization [24].

SI model is often applied to study on the epidemic dynamics at the early outbreak stages [25]. At the beginning, MERS in South Korea were mainly concentrated in three hospitals with obvious characteristics of community structure. In reality, the infection probability increases with the raise of contact time between infectious people and susceptible people, which must be highlighted in the epidemic spreading models. Edge weight is essential to describe the contact intimacy. In order to study the epidemic spreading characteristics and the optimal opportunity to take measures of MERS in South Korea, it is supposed that there is a linear relationship between contact strength and contact time. In this paper, spreading model is based on SI model with contact strength, and weighted network with community structure is based on BBV network model. The spreading characteristics are obtained by simulation according to the aforementioned models. Hence, they are verified by the real data in the South Korea MERS epidemic.

The remainder of this paper is organized as follows. Section 2 demonstrates the spreading model based on SI model in view of contact strength. Section 3 establishes the weighted network with community structure based on BBV model and analyzes the characteristics. Then, the spreading characteristics are studied to find the effective factors in Section 4, where the epidemic spreading process is divided into five stages. Section 5 studies the controlling measures (such as how to execute isolation and reduce the average weight of network) and the optimal opportunity to carry them out. Finally, case study based on MERS in South Korea is demonstrated in Section 6 to verify the models and the measure effects. Overall, both theoretical analysis and simulation results focus on the spreading characteristics and measure effects.

\section{Epidemic Spreading Model}

In this section, we suppose that the longer time the susceptible person contacts with the infectious ones, the larger probability the susceptible person will get infectious, at the beginning stage of the epidemic outbreak. In order to study the epidemic spreading process, we propose the spreading model based on contact strength and the computer simulation flowchart.

2.1. SI Model Based on Contact Strength. During the study of epidemic diffusion theory, the models are always based on some assumptions that the infectious unit is the node in the network and the epidemic can only spread through the links. The individuals are divided into 3 types: $S$ (Susceptible) means the healthy state which is likely to be infectious, $I$ (Infected) indicates the illness state which has already been infectious, and $R$ (Removed) signifies the immune state which has always been recovered or dead.

In terms of some epidemic bursting suddenly without valid control, such as SARS, H1N1 [26], and especially MERS, SI model is often used to study the spreading characteristics at the beginning period of diffusion process. Overall, prompt prevention measures would reduce the detrimental influence, which are of theoretical value and reality significance.

When the contact strength to an infectious person is $T_{1}$, the noninfectious probability of susceptible person is defined as

$$
(1-\lambda)^{T_{1} / T_{0}}
$$

Thus, when the contact strength to an infectious person is $T_{0}$, the infectious probability of susceptible person is defined as

$$
1-(1-\lambda)^{T_{0} / T_{0}}=\lambda
$$

One susceptible person contacts an infectious person with $T_{1}$ strength, then leaves for a while, and then contact the same infectious person with $T_{3}$ strength. If he does not get infection at the first time, he would seem as a healthy susceptible person at the second moment. That is, the two contacts are independent, and the noninfectious probability of susceptible person after second contact is

$$
(1-\lambda)^{T_{2} / T_{0}} \cdot(1-\lambda)^{T_{3} / T_{0}}=(1-\lambda)^{\left(T_{2}+T_{3}\right) / T_{0}} .
$$

If one susceptible point contacts two infectious points with strengths $T_{4}$ and $T_{5}$, respectively, the noninfectious probability of susceptible person after two contacts is

$$
(1-\lambda)^{\left(T_{4}+T_{5}\right) / T_{0}} .
$$

For susceptible point $i$, the infection probability $\lambda_{i}$ is

$$
\lambda_{i}=1-(1-\lambda)^{\sum_{j} T_{i j} / T_{0}},
$$

where $T_{i j}$ indicates the edge strength between point $i$ and infectious point $j$ and $\sum_{j} T_{i j}$ means the sum of edge strength between point $i$ and its adjacent infectious points.

$\left\langle\sum_{j} T_{i j}\right\rangle$ is defined as the average $\sum_{j} T_{i j}$ of each point in the whole network.

When the whole network is stable, the ratio of susceptible points is $s(t)$, and the ratio of infectious points is $i(t)$, then

$$
\begin{aligned}
s(t)+i(t) & =1, \\
\frac{\mathrm{d} i(t)}{\mathrm{d} t} & =\left[1-(1-\lambda)^{\left\langle\sum_{j} T_{j}\right\rangle / T_{0}}\right] i(t)[1-i(t)] .
\end{aligned}
$$

In the actual infection process, the longer time the susceptible person contacts the infectious person, the larger probability of susceptible person to get infection. In other 


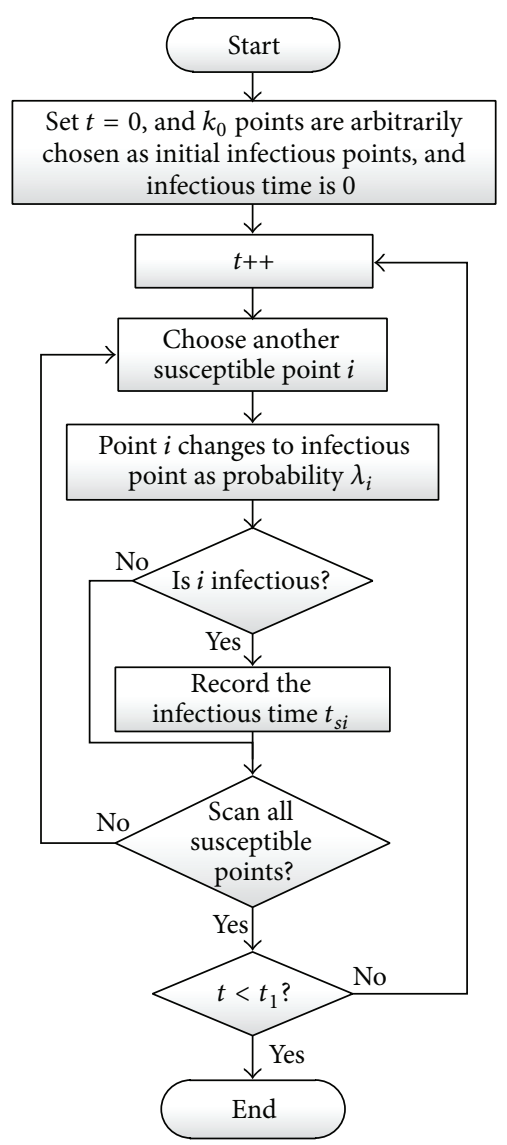

FIGURE 1: Flowchart of the spreading model simulation.

words, the edge strength between them is magnified. In conclusion, $T_{i j}$ is a formula $T_{i j}(t)$ that depends on time $t$.

For simplicity, it is assumed that there is a linear relationship between $T_{i j}(t)$ and $t$ :

$$
T_{i j}(t)=\alpha_{i j} \cdot t
$$

where $\alpha_{i j}$ indicates the coefficient of contact strength, $\alpha_{i j}<1$, $\langle\alpha\rangle$ means the average $\alpha_{i j}$, and $\langle k\rangle$ signifies the average point degree of network. Thus,

$$
\frac{\mathrm{d} i(t)}{\mathrm{d} t}=\left[1-(1-\lambda)^{(\langle k\rangle \cdot\langle\alpha\rangle t) / T_{0}}\right] i(t)[1-i(t)],
$$

where $a=(1-\lambda)^{(\langle k\rangle \cdot\langle\alpha\rangle) / T_{0}}$.

The solution is

$$
i(t)=\frac{1}{\left(i_{0}^{-1}-1\right) e^{-t+\ln ^{-1} a \cdot\left(a^{t}-1\right)}+1},
$$

where $i_{0}$ is the ratio of infectious points at time 0 , and when $t \rightarrow \infty, i(t)=1$.

2.2. Simulation of Spreading Model. According to the above spreading model, the simulation flowchart is shown in Figure 1.
In terms of susceptible point $i$, the infectious probability $\lambda_{i}$ is

$$
\lambda_{i}=1-(1-\lambda)^{\left(\sum_{j} \alpha_{i j} \cdot\left(t-t_{s j}\right)\right) / T_{0}},
$$

where $\alpha_{i j}$ is the edge weight, $t_{s j}$ is the infected moment for point $j$ and $T_{0}$ and $\lambda$ are the parameters related to the epidemic spreading characteristics.

During the simulation process, the time step length is always the same as $T_{0}$.

Epidemic spreads in the human-to-human network. After the construction of the epidemic spreading model based on the contact among people, we need to construct the network model. Due to the characteristics of people social contact, the weighted network model with community structure is essential to be constructed.

\section{Network Model}

This section establishes the weighted network with community structure and proposes the simulation process as the flowchart and then analyzes the sufficient conditions for the optimal community division. After that, the weight distribution and the community division characteristics are obtained [27].

3.1. Generation of Weighted Network with Community Structure. BBV network model is a weighted scale-free network model provided by Barrat et al. [14], which allows the dynamical evolution of weights during the growth of the system.

It is assumed that point $i$ is within the community $X, A_{i}$ indicates the strength of point $i, A_{i}^{I}$ means the inner strength of point $i$, and $A_{i}^{O}$ signifies the outer strength of point $i$; thus,

$$
\begin{aligned}
& A_{i}^{I}=\sum_{j \in X} \alpha_{i j}, \\
& A_{i}^{O}=\sum_{j \notin X} \alpha_{i j}, \\
& A_{i}=A_{i}^{I}+A_{i}^{O},
\end{aligned}
$$

where $\alpha_{i j}$ means the edge weight between point $i$ and point $j$; if $i$ and $j$ are disconnected, $\alpha_{i j}=0$. If point $i$ and point $j$ are in the same community, it is called edge weight inner the community, while if they are in two different communities, it is called edge weight outer the community.

Therefore, the evolution process of advanced BBV network is as follows.

Step 1 (initial setup). There are $S$ communities (i.e., $X_{1}, X_{2}$, $\ldots, X_{S}$ ) contributing to network $G$. The initial community has $m_{r_{0}}$ nodes (all $m_{r_{0}}$ are the same as $m$ ), connected by a small quantity of edges. And different communities are connected by several edges (without the inner links of points in the community), which constitutes the initial network. All initial edge weights are $\alpha_{0}$. 
Step 2 (adding points). Add a new point $i$ in each time step, and choose an arbitrary community (assumed as $X_{r}$ ) to join in. The new adding point is linked to the existing $m_{r}\left(m_{r} \leq\right.$ $m_{r_{0}}$ ) points in the community as the probability $p_{r}^{I}(j)$ (as shown in formula (1)) and linked to the existing $n(n<$ $\left.\sum_{s} m_{s_{0}}\right)$ points out of the community as the probability $p_{r}^{\mathrm{O}}(k)$ (as shown in formula (2)):

$$
\begin{aligned}
& p_{r}^{I}(j)=\frac{A_{j}^{I}}{\sum_{j \in X_{r}} A_{j}^{I}}, \\
& p_{r}^{O}(k)=\frac{A_{k}^{O}}{\sum_{k \in G} A_{k}^{O}} .
\end{aligned}
$$

Step 3 (weight change). The new adding edge is endued with an initial weight $\alpha_{0}$, which leads to the weight change of the inner community of conjoint point $j$ and the outer community of conjoint point $k$. The edge weights of the inner community are adapted to $\alpha_{j v}=\alpha_{j v}+\mu^{I} \alpha_{j v} / A_{i}^{I}$ (where $i$, $j$, and $v$ are located at different communities), and the edge weights of the outer community are adapted to $\alpha_{k l}=\alpha_{k l}+$ $\mu^{O} \alpha_{k l} / A_{k}^{O}$ (where $i$ and $k$ are located at different communities and $k$ and $l$ are located at different communities); $\mu^{I}$ and $\mu^{O}$ are the coefficients of weight enhancement.

Step 4 (weight normalization). After the construction of network, all the weights in the network are normalized. Assuming that the maximum edge weight of outer community is $\alpha_{m}^{O}$, the maximum edge weight of inner community is $\alpha_{m}^{I}$. Thus, the edge weights in the outer community of each point are normalized as $\alpha_{i}^{\mathrm{O}} / \alpha_{m}^{\mathrm{O}}$, and the edge weights in the inner community of each point are normalized as $\alpha_{i}^{I} / \alpha_{m}^{I}$.

Similar to the BBV network model, each community forms a BBV network; when $S$ is big, all points in the whole network are connected to the edges out of the community to construct a BBV network. According to the mean filed theory $[7,8]$ and BBV network model [14], both the inner weight distribution and outer weight distribution of community are in accordance with the power law.

According to the above four steps, the network meets all restrictions that are likely to be created as the simulation process of the weighted network with community structure in Figure 2.

3.2. Sufficient Conditions for Community Division. After the network generation, further research needs to analyze whether this kind of community division is the best. In this section, we study the sufficient condition to reach the optimal community division by analyses of modularity.

Salathé and Jones [23] proposed the representation method of modularity $Q$ in the weighted network:

$$
Q=\sum_{r=1}^{S}\left[e_{r r}-\left(h_{r}\right)^{2}\right]
$$

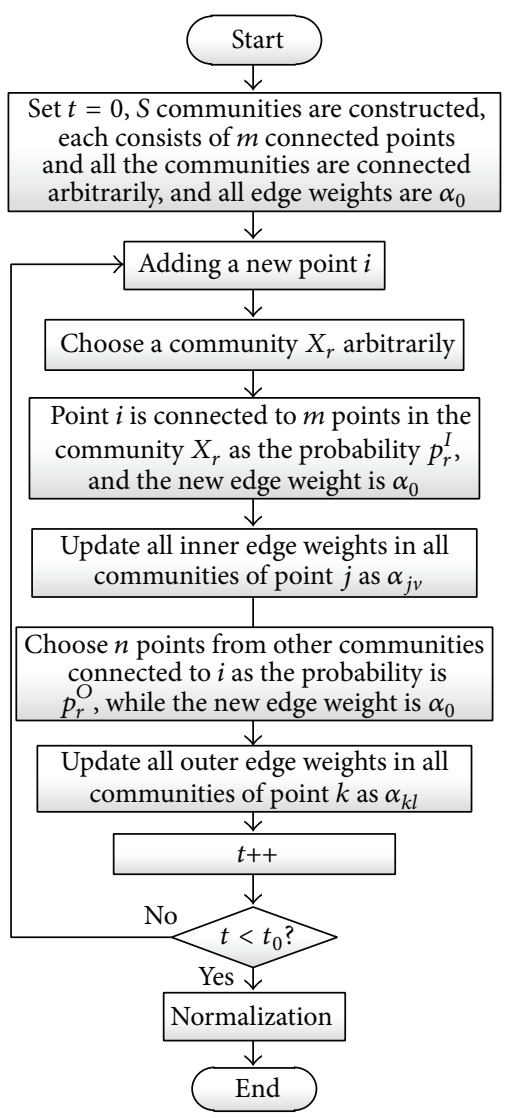

FIGURE 2: Flowchart of network model generation.

where

$$
\begin{aligned}
e_{r r} & =\frac{1}{2 A} \sum_{i, j \in X_{r}} \alpha_{i j}, \\
h_{r} & =\frac{1}{2 A} \sum_{i \in X_{r}} A_{i}, \\
A & =\frac{1}{2} \sum_{i, j \in G} \alpha_{i j} .
\end{aligned}
$$

In the formula, $A$ is the sum of all edge weights in network $G ; e_{r r}$ is the proportion of the sum edge weight of community $r$ occupying the edge weight sum of whole network; $h_{r}$ is the proportion of the sum point strength of community $r$ taken up in the whole network.

Then, it is essential to verify the former network model generated by four steps, where the modularity is $Q_{0}$. Two communities $X_{r}$ and $X_{s}$ are chosen arbitrarily from the network, and then one point $v$ in community $X_{s}$ is assigned to community $X_{r}$. Thus, the network modularity changes to $Q_{1}$, and the variation of modularity $\Delta Q$ is

$$
\begin{aligned}
\Delta Q= & Q_{0}-Q_{1} \\
= & {\left[e_{r r}-\left(h_{r}\right)^{2}+e_{s s}-\left(h_{s}\right)^{2}\right] } \\
& -\left[e_{r r}^{\prime}-\left(h_{r}^{\prime}\right)^{2}+e_{s s}^{\prime}-\left(h_{s}^{\prime}\right)^{2}\right]
\end{aligned}
$$




$$
\begin{aligned}
q_{0}= & e_{r r}-\left(h_{r}\right)^{2}+e_{s s}-\left(h_{s}\right)^{2}, \\
q_{1}= & e_{r r}^{\prime}-\left(h_{r}^{\prime}\right)^{2}+e_{s s}^{\prime}-\left(h_{s}^{\prime}\right)^{2}, \\
q_{1}= & {\left[\frac{2 A \cdot e_{r r}+2 A_{v, r}^{O}}{2 A}-\left(\frac{2 A \cdot h_{r}+A_{v}}{2 A}\right)^{2}\right] } \\
& +\left[\frac{2 A \cdot e_{s s}-2 A_{v}^{I}}{2 A}-\left(\frac{2 A \cdot h_{s}-A_{v}}{2 A}\right)^{2}\right], \\
\Delta Q= & q_{0}-q_{1} \\
= & \frac{A_{v}^{I}}{A}-\frac{A_{v, r}^{O}}{A}+2\left(\frac{A_{v}}{2 A}\right)^{2}+2\left(h_{r}-h_{s}\right) \frac{A_{v}}{2 A},
\end{aligned}
$$

where $A_{v, r}^{O}$ is the sum edge weight of the adjacent edges between point $v$ and inner points of community $X_{r}$, which names after the sum outer weight of community $r$ of point $v$, recorded as $\max _{r}\left(A_{v, r}^{O}\right)$.

During the network generation, all communities are at the same level, but when the network scale is bigger, generally speaking, $h_{r} \approx h_{s}$. Thus,

$$
\Delta Q \approx \frac{A_{v}^{I}}{A}-\frac{A_{v, r}^{O}}{A}+2\left(\frac{A_{v}}{2 A}\right)^{2} .
$$

During the whole process, it is assured that $\Delta Q \geq 0$.

When $A_{v}^{I} / A-A_{v, r}^{\mathrm{O}} / A>0$, it is known that $\Delta Q>$ $2\left(A_{v} / 2 A\right)^{2}>0$. Thus, if $A_{v}^{I}>A_{v, r}^{O}$, it is indubitable that the former community division methodology always gets the optimal result. If $A_{v}^{I}>A_{v}^{O}$ ( $v$ is the arbitrary point), it is surely satisfied to the optimal restrictions. During the network construction period, all points need to be connected to more inner points of community with higher contact strength and fewer outer points of community with lower contact strength.

After strict theoretical derivation and analyses, it is concluded that the sufficient condition to reach the optimal community division is $A_{v}^{I}>A_{v, r}^{O}$. Plenty of simulations should be done to analyze the characteristics.

\subsection{Characteristics of the Network}

3.3.1. Characteristics of the Edge Weight. After the weighted networks with community structure are generated, the characteristics of network are studied further. It focuses on the relationship of four characteristics and the community amount, or the coefficient of weight growth, separately. The four characteristics are the inner weight distribution of community, the outer weight distribution of community, the average inner weight of community, and the average outer weight distribution of community.

In Figure 3, there are 5000 points: $m=m_{0}=3, n=(S-$ 1) $\times m$, and $\mu^{I}=\mu^{\mathrm{O}}=1$.

It is demonstrated in Figure 3 as $\log$-log coordinate that the inner weight distributions of community obey the power law, which is $p(\alpha) \sim \alpha^{-\gamma}$. In terms of the inner weight distribution of community, $\gamma$ slightly increases with the rise
TABLE 1: Relationship of the average edge weight and the community amount.

\begin{tabular}{lccccc}
\hline$S$ & 50 & 100 & 150 & 200 & 250 \\
\hline Mean $\left(\alpha^{I}\right)$ & 0.111 & 0.182 & 0.206 & 0.216 & 0.238 \\
Mean $\left(\alpha^{O}\right)$ & 0.062 & 0.089 & 0.097 & 0.145 & 0.135 \\
\hline
\end{tabular}

of $S$; while in terms of the outer weight distribution of community, $\gamma$ is generally high when $S$ is large; anyway it is not strictly monotonous.

In Figure 4, there are 5000 points: $S=100, m=m_{0}=3$, $n=(S-1) \times m$, and $\mu^{I}=\mu^{O}=\mu$. The less the value of $\mu$ is, the larger the value of $\gamma$ is which related to the inner weight of the community or the outer weight of the community.

Figures 3 and 4 demonstrate that every BBV network is isolated independently with different communities, because the inner weight distribution of each community obeys the law power. However, Figures 3(a) and 4(a) are both the statistical results of the inner weight within the community, and thus there is deviation between the simulation result and the power-law distribution.

Supposing that mean $\left(\alpha^{I}\right)$ indicates the mean of inner weighs, mean $\left(\alpha^{O}\right)$ signifies the mean of outer weight.

In Table 1, there are 5000 points: $S=100, m=m_{0}=3$, $n=(S-1) \times m, \mu^{I}=\mu^{O}=1$.

With the increase of the community amount, the average inner weights of community get higher. Compared to the network with fewer communities, the average outer weight of community is relatively higher when the community amount is larger.

In Table 2, there are 5000 points: $S=100, m=m_{0}=3$, $n=(S-1) \times m$, and $\mu^{I}=\mu^{O}=\mu$.

It is demonstrated in Table 2 that with the growth of $\mu$, both the inner and the outer weight distributions of communities have the trend of decrease.

After the simulation, it is concluded that the weight distributions obey the power law and satisfy the reality well. Moreover, the power-law distribution characteristics rely on the parameters obviously, hence by control of which, any type of networks can be built.

3.3.2. Characteristics of the Community Structure. In this section, we will analyze the sufficient condition to satisfy the optimal community division and explain whether the network has a clear community structure by calculating the value of the modularity.

In Figure 5, there are 5000 points: $S=100, m=m_{0}=3$, $n=(S-1) \times m$, and $\mu^{I}=\mu^{O}=1$.

It is demonstrated that $A_{v}^{I}>\max _{r}\left(A_{v, r}^{O}\right)$, and each point meets the condition that $A_{v}^{I}>A_{v, r}^{O}$. In conclusion, the community division with this method is optimal.

In Table 3, there are 5000 points: $S=100, m=m_{0}=3$, $n=(S-1) \times m$, and $\mu^{I}=\mu^{O}=1$. And in Table 4, there are 5000 points: $S=100, m=m_{0}=3, n=(S-1) \times m$, and $\mu^{I}=\mu^{\mathrm{O}}=\mu$.

In reality, the value range of $Q$ in the network with community is $[0.3,0.7]$, and it is demonstrated that there is 
TABLE 2: Relationship of the average edge weights and the coefficient of weight growth.

\begin{tabular}{lccccccccc}
\hline$\mu$ & 0.5 & 0.55 & 0.6 & 0.65 & 0.7 & 0.8 & 0.9 & 1.0 & 1.5 \\
\hline Mean $\left(\alpha^{I}\right)$ & 0.2806 & 0.2848 & 0.2885 & 0.2493 & 0.2519 & 0.2117 & 0.1846 & 0.1816 & 0.1067 \\
Mean $\left(\alpha^{O}\right)$ & 0.1858 & 0.1929 & 0.1832 & 0.1431 & 0.1352 & 0.1206 & 0.1098 & 0.0894 & 0.0611 \\
\hline
\end{tabular}

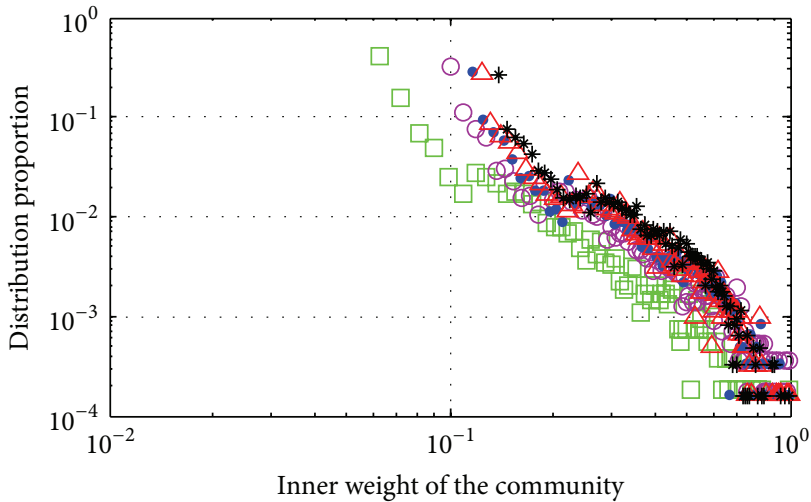

$\begin{array}{ll}\square 50 \text { communities } & \triangle 200 \text { communities } \\ -100 \text { communities } & * 250 \text { communities } \\ & 150 \text { communities }\end{array}$

(a) The relationship of inner weight distribution of community and the community amount

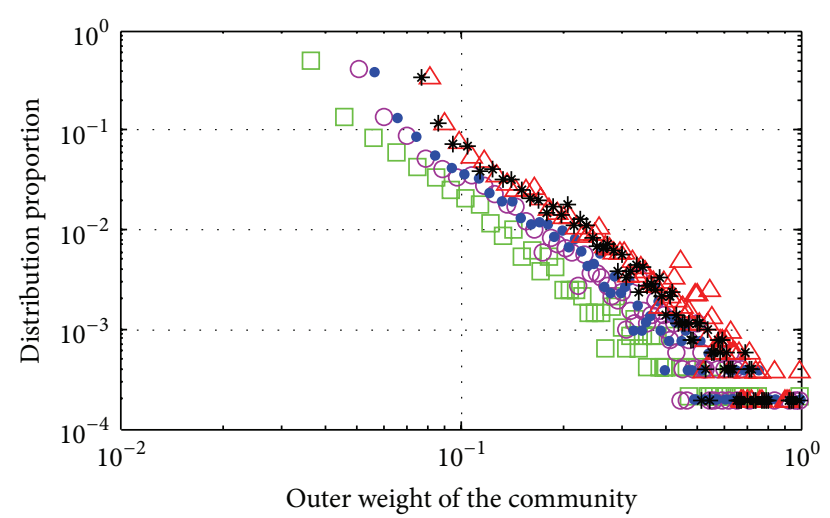

$\begin{array}{ll}\square 50 \text { communities } & \triangle 200 \text { communities } \\ -100 \text { communities } & * 250 \text { communities } \\ -150 \text { communities } & \end{array}$

(b) The relationship of outer weight distribution of community and the community amount

FIGURE 3: Relationship of weight distribution and the community amount.

TABLE 3: Relationship of $Q$ value and community amount.

\begin{tabular}{cccccc}
\hline$S$ & 50 & 100 & 150 & 200 & 250 \\
\hline$Q$ & 0.6439 & 0.6728 & 0.6950 & 0.6147 & 0.6640 \\
\hline
\end{tabular}

obvious community structure in the network from Tables 3 and 4.

\section{Characteristics of Epidemic Spreading}

This section studies the specific characteristics of the epidemic spreading based on the aforementioned models, including the infection proportion of epidemic spreading in different stages, and the relationship of transmission rate and the community amount, or the average network weight, or the strength of the initial infectious point, separately.

\subsection{Influence of Community Amount to Epidemic Spread-} ing. It is defined that the epidemic transmission rate $\mathrm{d} \eta$ means the increase rate of the infection proportion. During the simulation process, the transmission rate $\varphi(t)$ is defined as the amount of new infectious points in each simulation time step.

According to Figure 6, in terms of the infection proportion variation curve for 200 communities, given 4 infection proportions, namely, $\eta\left(t_{1}\right), \eta\left(t_{2}\right), \eta\left(t_{3}\right), \eta\left(t_{4}\right)$, we know that $\eta(0)<\eta\left(t_{1}\right)<\eta\left(t_{2}\right)<\eta\left(t_{3}\right)<\eta\left(t_{4}\right)<\eta(\infty)$, corresponding to $t_{1}, t_{2}, t_{3}, t_{4}\left(t_{1}<t_{2}<t_{3}<t_{4}\right)$, respectively. $\varphi\left(t_{2}\right)$ and $\varphi\left(t_{3}\right)$ are the transmission rate at $t_{2}$ and $t_{3}$, given $\varphi_{1}=\min \left(\varphi\left(t_{2}\right), \varphi\left(t_{3}\right)\right)$, respectively. Therefore, the epidemic spreading process can be divided into 5 stages.

Stage 1 (the initial stage of spreading). $\eta(0) \leq \eta(t)<\eta\left(t_{1}\right)$, where $\eta(t)$ is small, and the transmission rate is slow while the infection people are gathered in a small range.

Stage 2 (the initial stage of outbreak). $\eta\left(t_{1}\right) \leq \eta(t)<\eta\left(t_{2}\right)$, and the transmission rate increases constantly but $\varphi(t)<$ $\varphi\left(t_{2}\right)$.

Stage 3 (the middle stage of outbreak). $\eta\left(t_{2}\right) \leq \eta(t)<\eta\left(t_{3}\right)$, and the infection rate is larger than some value $\varphi(t) \geq \varphi_{1}$.

Stage 4 (the end stage of outbreak). $\eta\left(t_{3}\right) \leq \eta(t)<\eta\left(t_{4}\right)$, and the transmission rate decreases constantly but $\varphi(t)<\varphi\left(t_{3}\right)$.

Stage 5 (the end stage of spreading). $\eta(t)>\eta\left(t_{4}\right)$, the infection proportion is larger than some value, and at this stage, the whole network is nearly infectious.

In comparison with the multiple simulations, the relationships between the infection proportion with different community amounts and the increase of time step length are shown in Figure 6.

In Figure 6, there are 5000 points, while the average inner weights of community are normalized as 0.1 , and the average outer weights of community are normalized as 0.05 .

The four curves are shown in Figure 6, when the average weights are analogous, the four curves have similar variation trend at Stages 2 to 5 , and these four stages are irrelative to 


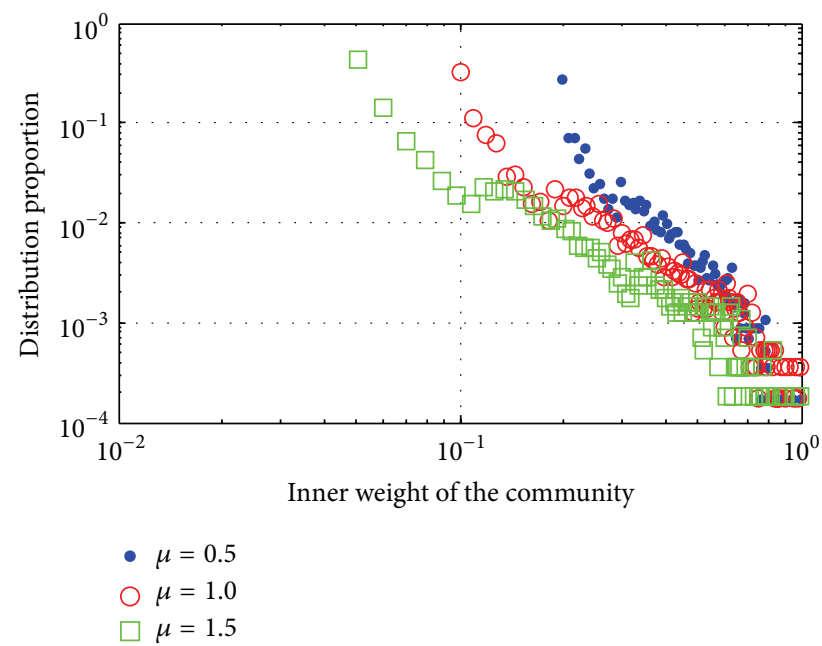

(a) Relationship of inner weight distribution of community and the coefficient of weight growth

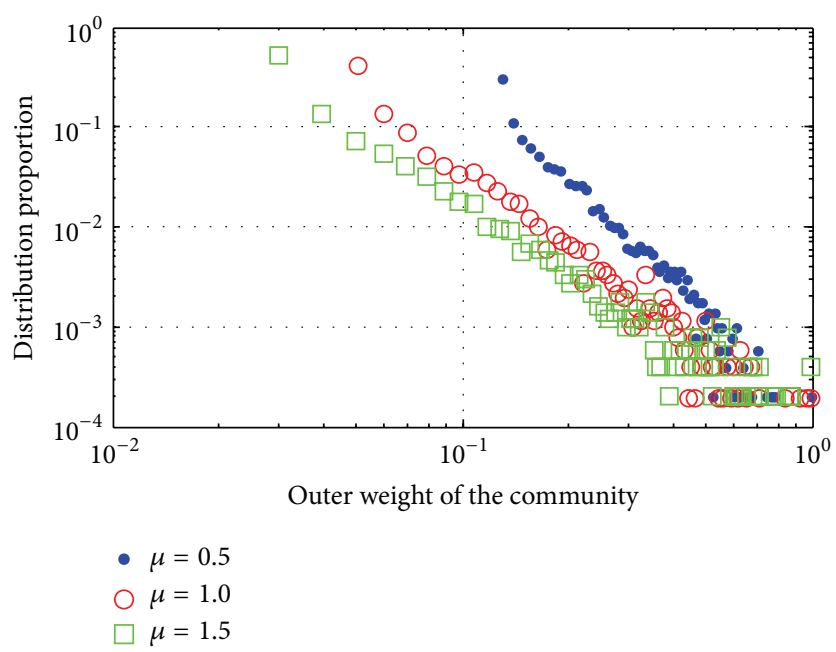

(b) Relationship of outer weight distribution of community and the coefficient of weight growth

FIGURE 4: Relationship of community weight distribution and the coefficient of weight growth.

TABLE 4: Relationship of $Q$ value and the coefficient of weight growth.

\begin{tabular}{cccccccccc}
\hline$\mu$ & 0.50 & 0.55 & 0.60 & 0.65 & 0.70 & 0.80 & 0.90 & 1.0 & 1.5 \\
\hline$Q$ & 0.6090 & 0.6054 & 0.6158 & 0.6376 & 0.6554 & 0.6358 & 0.6281 & 0.6728 & 0.6397 \\
\hline
\end{tabular}

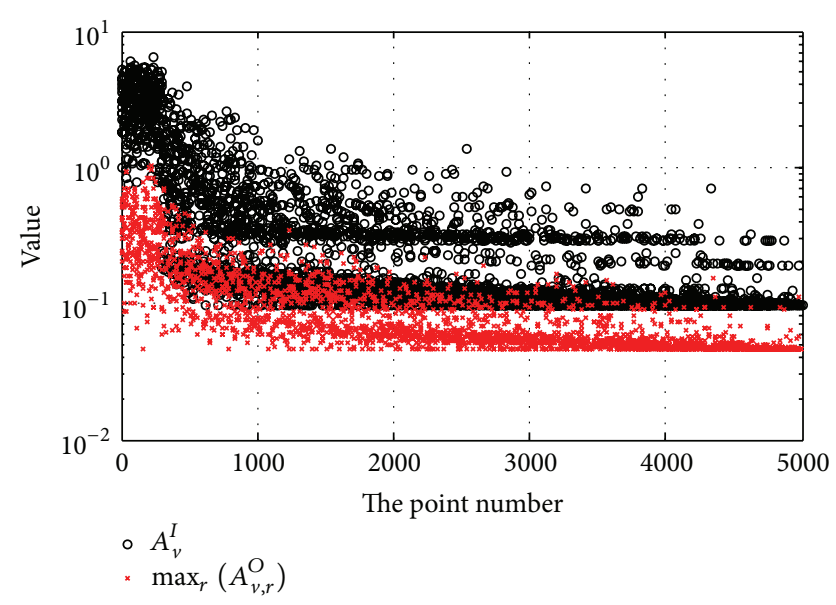

FIgURE 5: Comparison graph of $A_{v}^{I}$ and $\max _{r}\left(A_{v, r}^{O}\right)$.

the community amount without any control measurements. Compared to curves 2 and 4, it is found that there is randomness in the initial spreading stage related to the sum weight of initial infectious points.

\subsection{Influence of the Average Weight to Epidemic Spreading. In} Figure 7, there are 5000 points and $S=100$.

The later four stages are related to the average weight of the whole network, and the bigger the mean is, the larger the maximum infection proportion would be, and the transmission rate reaches the highest at the initial stage of

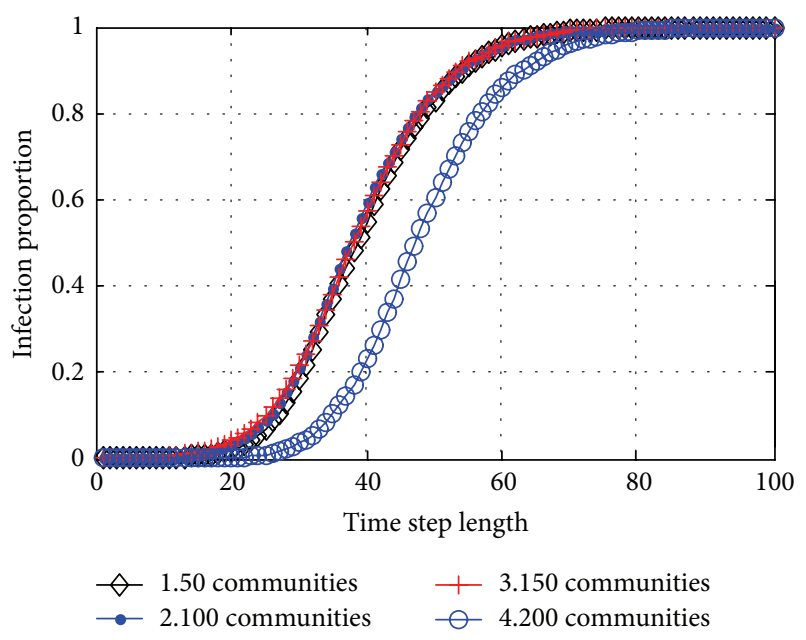

FIGURE 6: Simulation graph of infection conditions with different community amounts.

outbreak. It is the middle stage of outbreak that the average weight has most effects on; therefore, reducing the weights in the network can postpone the outbreak to some extent.

The parameters related to the infection ability affect the five stages. People barely have any effective measures to master the epidemic during the initial stage of spreading. This paper focuses on the control of large-scale epidemic spreading and the effective measures of nodes isolation and 


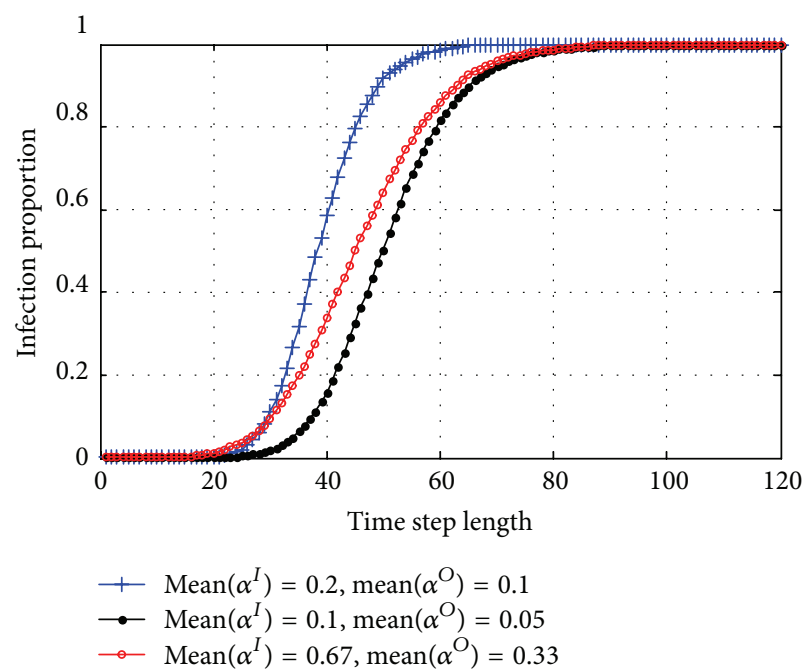

(a) The relationship of the infection proportion and the average weights

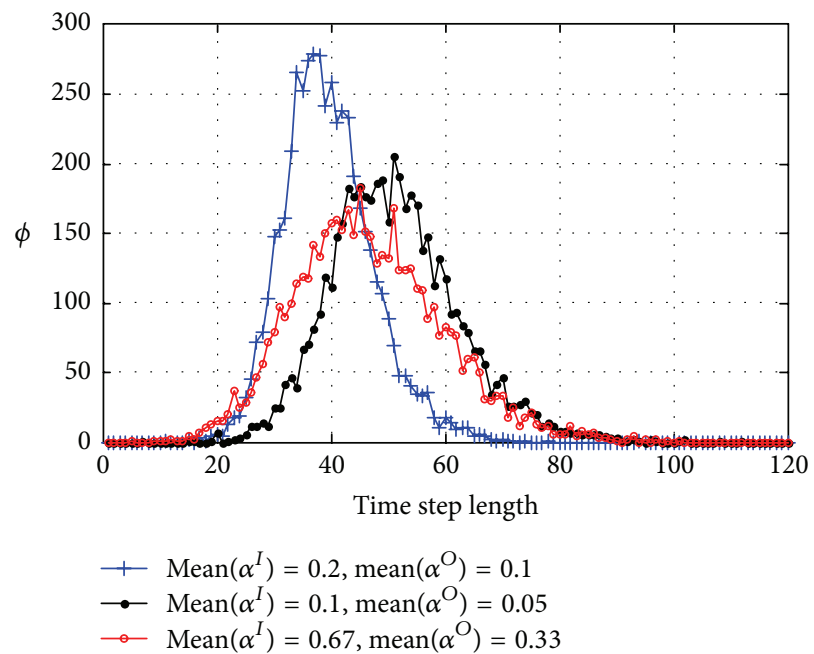

(b) The relationship of the infection rate and the average weights

FIGURE 7: Relationship between the average weights and the infection proportion or the infection rate.

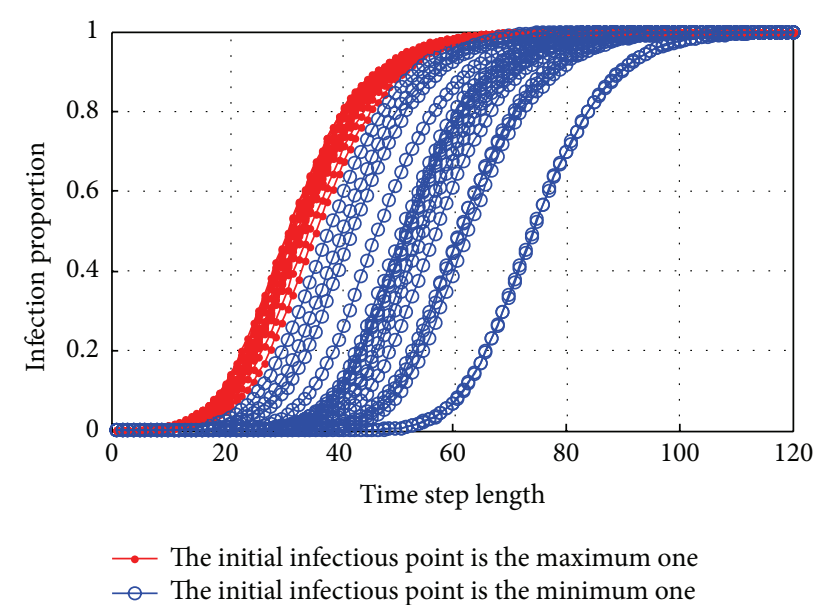

FIGURE 8: Relationship of infection proportion and the strength of initial infectious point.

edge weights, although the impact of these parameters on epidemic spreading is not further studied in detail.

\subsection{Influence of Initial Infection Strength to Epidemic Spread-} ing. In Figure 8, there are 5000 points, $S=100$, mean $\left(\alpha^{I}\right)=$ 0.1 , and mean $\left(\alpha^{\mathrm{O}}\right)=0.05$. It has been simulated 16 times when the strength of the initial infectious point is the maximum and another 16 times when the strength of the initial infectious point is the minimum.

Figure 8 shows that when the strength of initial infectious point is large, the continuous time at the first stage of epidemic spreading is shorter and more centralized after several times of simulation; when the strength of initial infectious point is small, the continuous time is longer and distributed more randomly.
Through the simulation, it is concluded that the epidemic transmission rate has explicit relationship with the average weight of the network other than the community amount, especially at the later four stages of epidemic spreading. There is randomness at the first stage of epidemic spreading, while the larger the initial infectious point strength is, the less the randomness will be. Moreover, the first stage duration time is longer when the strength of the initial infectious point is less.

\section{Measures to Control Epidemic Spreading}

5.1. Measures to Isolate the Infectious Points. In reality, at the very beginning of the epidemic spreading process, it is hard to attract the public attention due to the minority of infectors [28]. However, we hope to control the epidemic at the initial stage of epidemic outbreak. Assuming there is a constant parameter related to the infection ability, it is concluded after several simulations that

(1) from the initial stage of outbreak to the end stage of epidemic spreading, the infection proportion is stable around the value $i_{\infty}\left(i_{\infty} \leq 1\right)$;

(2) the stable $i_{\infty}\left(i_{\infty} \leq 1\right)$ is related to $t_{0}$ and $\lambda$.

The simulation result is demonstrated as in Figure 9. There are 5000 points, $S=100, m=m_{0}=3, n=(S-1) \times m=$ $6, \mu^{I}=\mu^{\mathrm{O}}=1, T_{0}=1$, and $\lambda=0.1$. The amount of the initial infectious points is 10 , and there are simulation curves at each $t_{0}$.

It is defined that the extreme isolation delay $t_{0}$ is signed as $m_{t 0}$ which makes the final stable infection proportion $i_{\infty}$ satisfy $i_{\infty} \leq 0.9$, in terms of the infection probability $\lambda$ at $T_{0}=1$.

In Table 5, there are 5000 points, $S=100, m=m_{0}=3$, $n=(S-1) \times m=6$, and $\mu^{I}=\mu^{O}=1$.

This section focuses on the influence of isolation delay on epidemic spreading and obtains that timely isolation can 


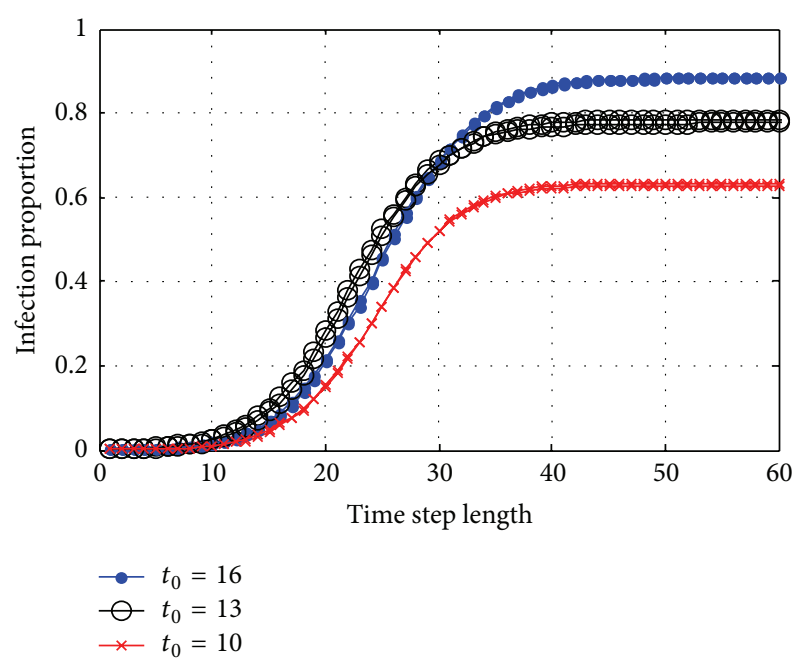

FIGURE 9: Relationship of $\eta$ and the step length with different $t_{0}$.

TABLE 5: The relationship of $m_{t_{0}}$ and $\lambda$.

\begin{tabular}{lcccccccc}
\hline$\lambda$ & 0.1 & 0.2 & 0.3 & 0.4 & 0.5 & 0.6 & 0.7 & 0.8 \\
\hline$m_{t_{0}}$ & 16 & 11 & 9 & 8 & 7 & 6 & 5 & 4 \\
\hline
\end{tabular}

reduce the final infection proportion $i_{\infty}$, which indicates that there is direct relationship between $i_{\infty}$ and the isolation delay.

5.2. Measures to Reduce the Average Weight of Network. In reality, during the control process at the initial stage of the infectious epidemic, it exists infectivity for some viruses without any explicit symptoms [29]. Coupled with the isolation efficiency, it is difficult to put all infectious points isolated at the initial stage; in other words, it is hard to make $t_{0}$ less. Accordingly, if only taking measurement as point isolation, the epidemic infection proportion $i_{\infty}$ eventually reaches a large value, which is not conducive to control the epidemic spreading.

If the isolation measures are not taken in time, it is also required to control the whole network by other measures in order to better control the epidemic spreading. In reality, it is often acceptable that the healthy person should enhance the self-protection conscience and cut down the opportunities to contact with the infectious ones, which equals reducing the average weight of points and canceling some edges in the network model [30].

Nowadays, the widely acceptable methods to control the whole network include keeping away from public places and minimizing direct contact with each other as much as possible [31, 32]. However, if measures are carried out too early or too much, it will change people's living habits, increase the economic loss, and cause large-scale social panic, but if it is too late, it will lose the best opportunity to control the epidemic spreading.

It is demonstrated in Figure 7 that it can only postpone the epidemic outbreak other than cut down the final spreading proportion by reducing the average weight of the network. However, it is not useful to lessen the average weight of the

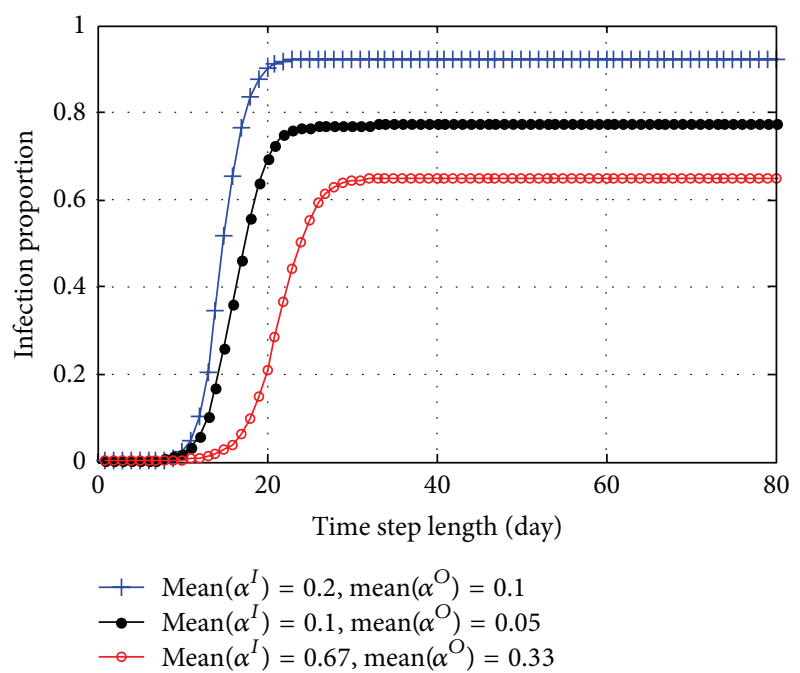

FIGURE 10: Relationship of infection proportion and time step length after points isolation.

network at Stage 1 (the initial stage of epidemic spreading) but to make point isolation. At Stage 2 (the initial stage of outbreak), if the isolation delay $t_{0}$ cannot meet the requirements even when the infection proportion becomes $i_{\infty}$, it is essential to take measures to decrease the average weight of the network.

Figure 10 depicts the influence of different network average weights have on $i_{\infty}$ after the point isolation.

In Figure 10, there are 5000 points, $S=100, m=m_{0}=3$, $n=(S-1) \times m=6, T_{0}=1, \lambda=0.6$, and $t_{0}=6$. When the isolation measures are taken and the isolation delay $t_{0}$ is constant, the less the average weight of the network is, the smaller the value of $i_{\infty}$ would be.

In reality, once the epidemic breaks out, the isolation measures should be taken; then, we can analyze the characteristics of epidemic spreading by statistical method and study the isolation delay. It is necessary to take some measures to cut down the average weight of the network at Stage 2 (the initial stage of outbreak). When it comes to Stage 3 (the middle stage of outbreak), it is essential to decrease the average weight of the network, in order to make it come into Stage 4 (the end stage of outbreak) and Stage 5 (the end stage of spreading) as soon as possible.

\section{Case Study on MERS}

6.1. Case Study of Epidemic Spreading Characteristics. Epidemic spreading model based on contact strength was proposed in Section 2, while weighted network model with community structure was proposed in Section 3. However, real data is essential to verify whether the epidemic spreading result based on this model is persuasive.

From the first MERS infection case that was diagnosed definitely in South Korea, there have been 166 people infected until 20 June 2015. The change trend of infection population during these 31 days is shown in Figure 11. 
It is not until June 7th when the South Korea government actually issued a response to MERS; in consequence, the 19 days from May 20th to June 7th should be regarded as the initial stage of the epidemic outbreak without interference measures, and the infection population is 87 . The epidemic in this period mainly occurred in 3 hospitals, namely, Pyongtaek Mary Hospital, Konyang University Hospital, and Daejeon Hospital. They are considered as 3 communities $(S=3)$, and the sum of points is regarded as the total amount of contact points in epidemic spreading network with the three communities. Then the fitting result of the simulation data and the real-world data are demonstrated in Figure 12, where the infection proportion $\eta$ means the ratio of infectious points in the total amount of points in the network.

From May 20th to June 7th, the population is about 5001000 in the contact network of the three hospitals. Hence, the simulation parameters in Figure 12 are as follows: in terms of the network model, the total amount of points is $600, S=3$, $m=m_{0}=3, n=(S-1) \times m=6, \mu^{I}=0.2$, and $\mu^{O}=0.6$; in terms of the epidemic spreading model, $T_{0}=2.4, \lambda=0.1$, the initial amount of infectious points is 1 . The real data curve is drawn by the MERS infectious population in South Korea divided into 600 .

Overall, due to the fitting result in Figure 12, the real initial stage of epidemic outbreak can be explained well by the network model and the epidemic spreading model proposed in this paper.

In this section, the accuracy of models in this paper is verified by comparison of the real data.

6.2. Case Study of Control Measures. Figure 13 shows the comparison about the simulation result of infection proportion change with the increase of time step length and the real data after June 7 th when the isolation measures were taken by South Korea government.

In Figure 13, there are 600 points, $S=3, m=m_{0}=3$, $n=(S-1) \times m=6, \mu^{I}=0.2$, and $\mu^{O}=0.6$, and in the epidemic spreading model, $T_{0}=2.4, \lambda=0.1$, the initial infection population is 1 , and the isolation measure is taken at the 19 th step, $t_{0}=4$. The real data curve is drawn by the MERS infectious population divided into 600 .

It is demonstrated in Figure 13 that the curve can fit the real infection proportion statistics curve when the isolation delay $t_{0}=4$. However, the South Korea government took measure at Stage 2 (the initial stage of outbreak) actually, which was a little later. The simulation result is shown as in Figure 14 if the measures were taken at Stage 1 (the initial stage of epidemic spreading).

In Figure 14, the network model and epidemic spreading model are the same as Figure $13, t_{0}=4$, but the isolation measure is taken at the first step. The 6 simulation result curves are the 6 results with maximum $i_{\infty}$ among the 100 times simulation.

Figure 14 demonstrates that if we could take measures at the very beginning of epidemic spreading, the final infection proportion $i_{\infty}$ would be reduced significantly even with the same isolation delay. However, due to the abruptness of epidemic outbreak, it is difficult to master control at

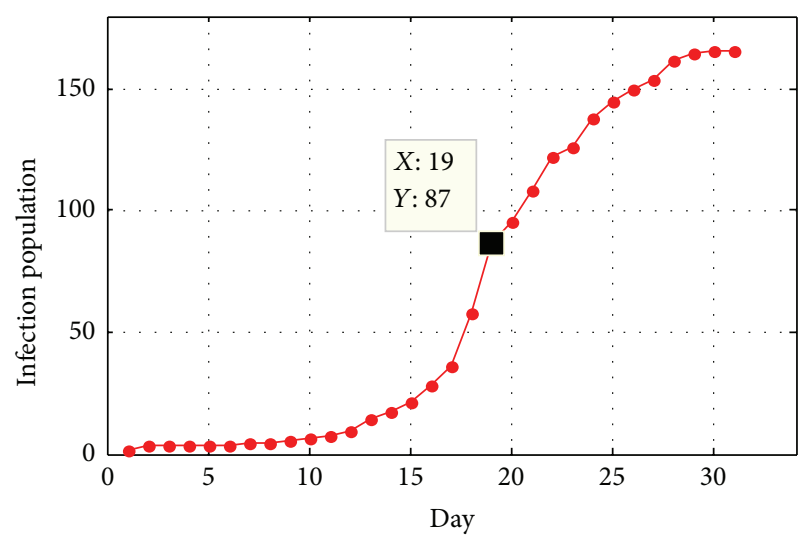

FIgURE 11: Statistics of MERS infection population in South Korea.

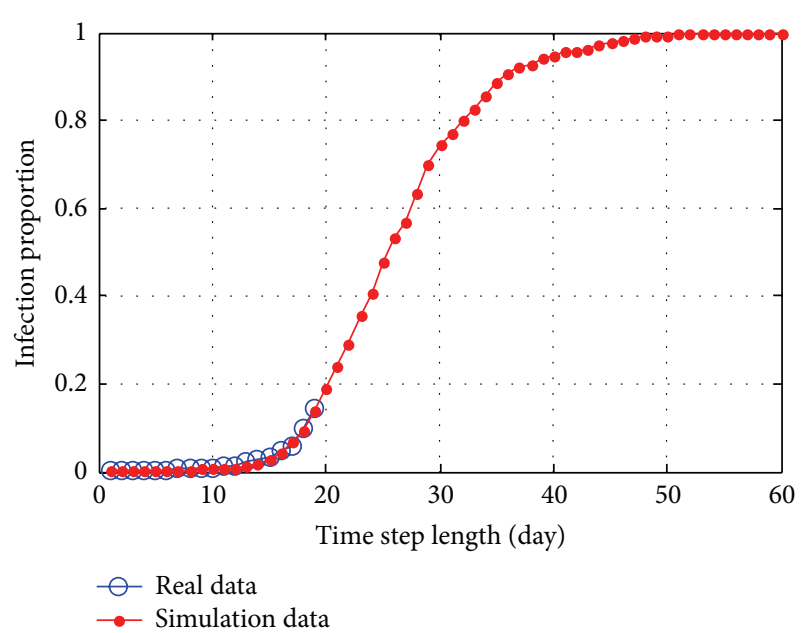

FIGURE 12: Fitting result of the simulation infection proportion and the real-world data.

the early stage. Consequently, it is essential to reduce the average weight of the network by some auxiliary measures, for example, dissolving some organizations with evident community structure (such as schools), decreasing the stay time in public places, or canceling some public activities.

\section{Conclusion}

MERS viruses are spreading swiftly at the very beginning period among the intimate human-to-human contact communities. The control measurements and the optimal opportunity to take action are proposed to study the spreading characteristics. Assuming that the contact strength has a linear relationship with time, the epidemic spreading model is established based on the SI model, which is the foundation of analytical solution research. The weighted network model with community structure is constructed on the basis of the BBV network model, deducing that the generated network meets the sufficient conditions for the optimal community division. The simulation results show that the weight distributions within the community and among the communities both obey the power-law distribution. 


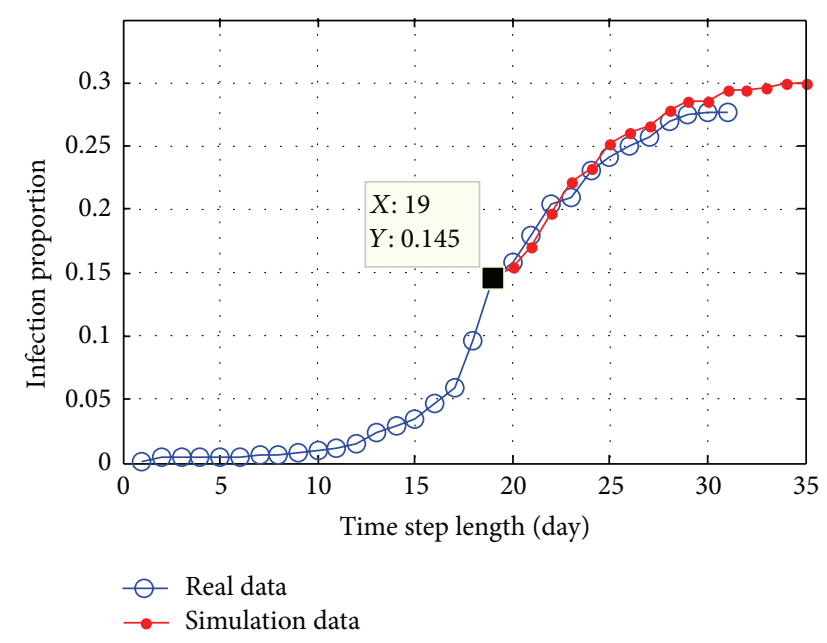

FIGURE 13: Comparative graph of simulation result and real data after the measures.

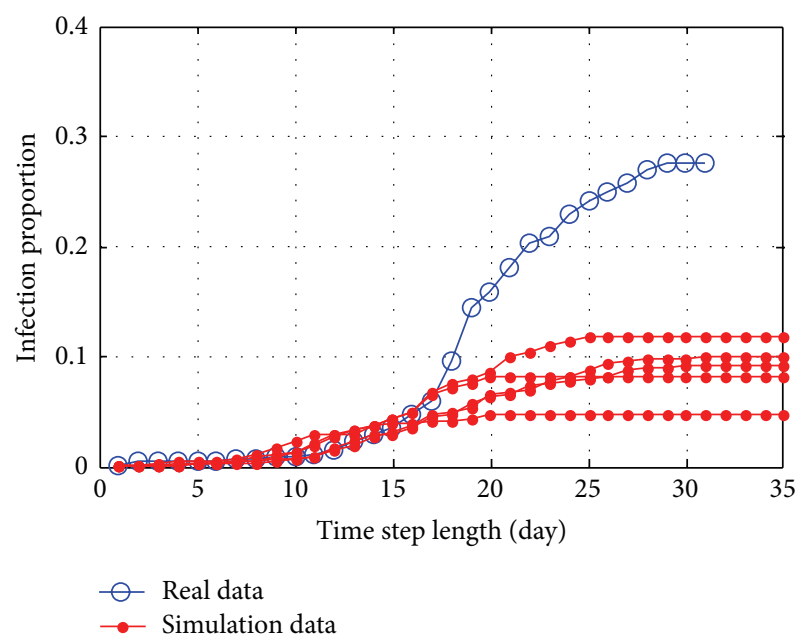

FIGURE 14: Simulation result when the isolation measures are taken at Stage 1.

According to the epidemic spreading network model, this research focuses on the characteristics of virus transmission. Hence, the epidemic spreading process would be divided into five stages: the initial stage of spreading, the initial stage of outbreak, the middle stage of outbreak, the end stage of outbreak, and the end stage of spreading. Then the simulation results show that the duration time of Stage 1 is related to the sum weight of the initial infection nodes, while those of Stages 2 to 5 are only related to the average weight instead of the community amount. However, without isolation measures, the decrease of the average weight only postpones spreading without interdicting it.

There is always a time delay before patients are isolated; thus, we study on the effects and optimal opportunity to master the epidemic spreading by two approaches: the point isolation and the average weight of network reduction. In conclusion, shorter time delay would reduce the final infection proportion, and with the same time delay, the decline of the average weight would further reduce the final infection proportion. The simulation data matches the real data of MERS in South Korea well. Therefore, the measures are adopted as follows: firstly isolate the infectious points as soon as possible; if the infection proportion is higher than the setting threshold, then the average weight should be cut down to the corresponding value (e.g., canceling public activities, dissolving some organizations, and keeping away from the crowded places).

The models in this paper are also appropriate for the diffusion of terrorist forces or evil forces with intimate community structures. Since the proposed epidemic spreading model is based on the SI model, it is hard to simulate the condition in which infectious people recover or die after a period of epidemic outbreak. Moreover, it is supposed that there is a linear relationship between contact strength and contact time, which cannot mimic the reality very well. In the future, we will study on different diseases with different epidemic spreading models on the proposed weighted network model with community structure after the period of large-scale infection or immunization.

\section{Conflict of Interests}

The authors declare that there is no conflict of interests regarding the publication of this paper.

\section{Acknowledgment}

This work was supported in part by the National Natural Science Foundation of China under Grant nos. 71331008 and 71501182 .

\section{References}

[1] R. M. Anderson, R. M. May, and B. Anderson, "The mathematics of infection.(Book reviews: infectious diseases of humans. dynamics and control)," Science, vol. 254, no. 2, pp. 591-592, 1991.

[2] N. Bailey, "The mathematical theory of infectious diseases and its applications," Immunology, vol. 34, no. 5, pp. 955-956, 1978.

[3] H. W. Hethcote, "The mathematics of infectious diseases," SIAM Review, vol. 42, no. 4, pp. 599-653, 2000.

[4] M. E. Newman, "The structure and function of complex networks," SIAM Review, vol. 45, no. 2, pp. 167-256, 2003.

[5] C. Li, W. Hu, and T. Huang, "Stability and bifurcation analysis of a modified epidemic model for computer viruses," Mathematical Problems in Engineering, vol. 2014, Article ID 784684, 14 pages, 2014.

[6] C. Fraser, C. A. Donnelly, S. Cauchemez et al., "Pandemic potential of a strain of influenza A (H1N1): early findings," Science, vol. 324, no. 5934, pp. 1557-1561, 2009.

[7] M. E. J. Newman, S. Forrest, and J. Balthrop, "Email networks and the spread of computer viruses," Physical Review E, vol. 66, no. 3, Article ID 035101, 2002.

[8] R. Pastor-Satorras and A. Vespignani, "Epidemic dynamics in finite size scale-free networks," Physical Review E, vol. 65, no. 3, Article ID 035108, 2002.

[9] Y. Wang, D. Chakrabarti, C. Wang, and C. Faloutsos, "Epidemic spreading in real networks: an eigenvalue viewpoint," 
in Proceedings of the 22nd International Symposium on Reliable Distributed Systems (SRDS '03), pp. 25-34, IEEE, Florence, Italy, October 2003.

[10] D. Chakrabarti, Y. Wang, C. Wang, J. Leskovec, and C. Faloutsos, "Epidemic thresholds in real networks," ACM Transactions on Information and System Security, vol. 10, no. 4, pp. 1311-1326, 2008.

[11] P. Erdös, “On the structure of linear graphs," Israel Journal of Mathematics, vol. 1, no. 3, pp. 156-160, 1963.

[12] D. J. Watts and S. H. Strogatz, "Collective dynamics of 'smallworld' networks," Nature, vol. 393, no. 6684, pp. 440-442, 1998.

[13] A.-L. Barabási and R. Albert, "Emergence of scaling in random networks," Science, vol. 286, no. 5439, pp. 509-512, 1999.

[14] A. Barrat, M. Barthélemy, and A. Vespignan, "Modeling the evolution of weighted networks," Physical Review E, vol. 70, no. 6, Article ID 066149, 12 pages, 2004.

[15] M. Girvan and M. E. Newman, "Community structure in social and biological networks," Proceedings of the National Academy of Sciences of the United States of America, vol. 99, no. 12, pp. 7821-7826, 2002.

[16] P. M. Gleiser and L. Danon, "Community structure in jazz," Advances in Complex Systems, vol. 6, no. 4, pp. 565-573, 2003.

[17] M. E. J. Newman, "Communities, modules and large-scale structure in networks," Nature Physics, vol. 8, no. 1, pp. 25-31, 2012.

[18] J.-P. Zhang and Z. Jin, "Epidemic spreading on complex networks with community structure," Applied Mathematics and Computation, vol. 219, no. 6, pp. 2829-2838, 2012.

[19] B. Serrour, A. Arenas, and S. Gómez, "Detecting communities of triangles in complex networks using spectral optimization," Computer Communications, vol. 34, no. 5, pp. 629-634, 2011.

[20] D. Zhang, F. Xie, Y. Zhang, F. Dong, and K. Hirota, "Fuzzy analysis of community detection in complex networks," Physica A: Statistical Mechanics and its Applications, vol. 389, no. 22, pp. 5319-5327, 2010.

[21] Z. Liu and B. Hu, "Epidemic spreading in community networks," Europhysics Letters, vol. 72, no. 2, pp. 315-321, 2005.

[22] T. Smieszek, L. Fiebig, and R. W. Scholz, "Models of epidemics: When contact repetition and clustering should be included," Theoretical Biology and Medical Modelling, vol. 6, article 11, 2009.

[23] M. Salathé and J. H. Jones, "Dynamics and control of diseases in networks with community structure," PLoS Computational Biology, vol. 6, no. 4, pp. 387-395, 2010.

[24] C.-H. Li, C.-C. Tsai, and S.-Y. Yang, "Analysis of epidemic spreading of an SIRS model in complex heterogeneous networks," Communications in Nonlinear Science and Numerical Simulation, vol. 19, no. 4, pp. 1042-1054, 2014.

[25] Y. Wang, G. Xiao, J. Hu, T. H. Cheng, and L. Wang, "Imperfect targeted immunization in scale-free networks," Physica A: Statistical Mechanics and Its Applications, vol. 388, no. 12, pp. 2535-2546, 2009.

[26] W. Zhao, J. Li, and X. Meng, "Dynamical analysis of SIR epidemic model with nonlinear pulse vaccination and lifelong immunity," Discrete Dynamics in Nature and Society, vol. 2015, Article ID 848623, 10 pages, 2015.

[27] P. Mundur, M. Seligman, and G. Lee, "Epidemic routing with immunity in Delay Tolerant Networks," in Proceedings of IEEE Military Communications Conference (MILCOM '08), pp. 1-7, San Diego, Calif, USA, November 2008.

[28] K. Gao and D. Hua, "Effects of immunity on global oscillations in epidemic spreading in small-world networks," Physics Procedia, vol. 3, no. 5, pp. 1801-1809, 2010.
[29] C.-Y. Xia, Z. Wang, J. Sanz, S. Meloni, and Y. Moreno, "Effects of delayed recovery and nonuniform transmission on the spreading of diseases in complex networks," Physica A: Statistical Mechanics and its Applications, vol. 392, no. 7, pp. 1577-1585, 2013.

[30] N. Gupta, A. Singh, and H. Cherifi, "Community-based immunization strategies for epidemic control," in Proceeding of the 7th IEEE International Conference on Communication Systems and Network, pp. 1-6, Chengdu, China, 2015.

[31] X. Fu, M. Small, D. M. Walker, and H. Zhang, "Epidemic dynamics on scale-free networks with piecewise linear infectivity and immunization," Physical Review E, vol. 77, no. 3, Article ID 036113, pp. 420-427, 2008.

[32] S. Cauchemez, A. Bhattarai, T. L. Marchbanks et al., "Role of social networks in shaping disease transmission during a community outbreak of 2009 H1N1 pandemic influenza," Proceedings of the National Academy of Sciences of the United States of America, vol. 108, no. 7, pp. 2825-2830, 2011. 


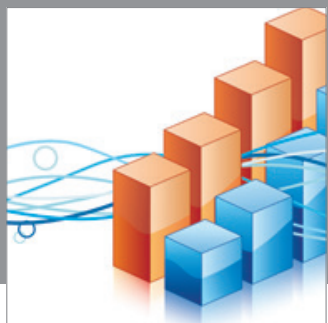

Advances in

Operations Research

mansans

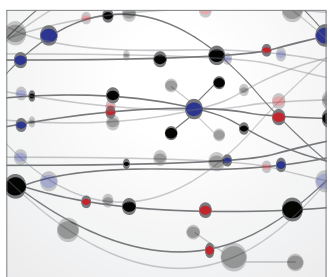

The Scientific World Journal
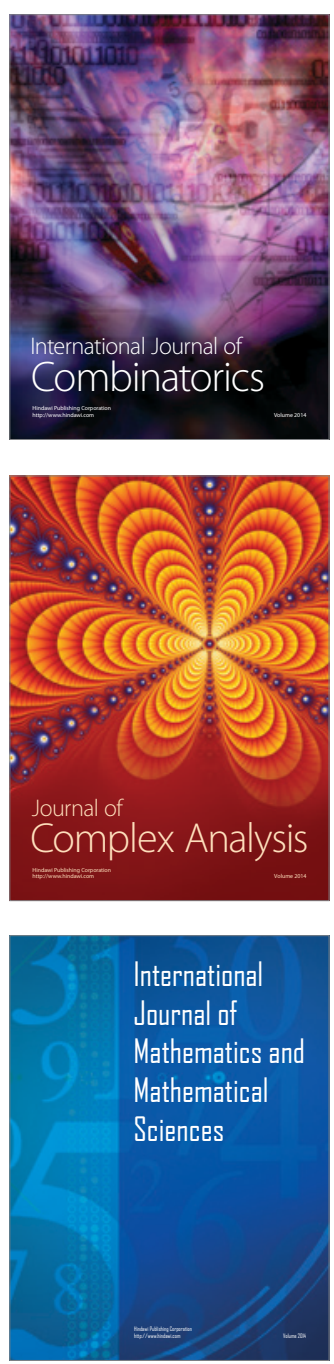
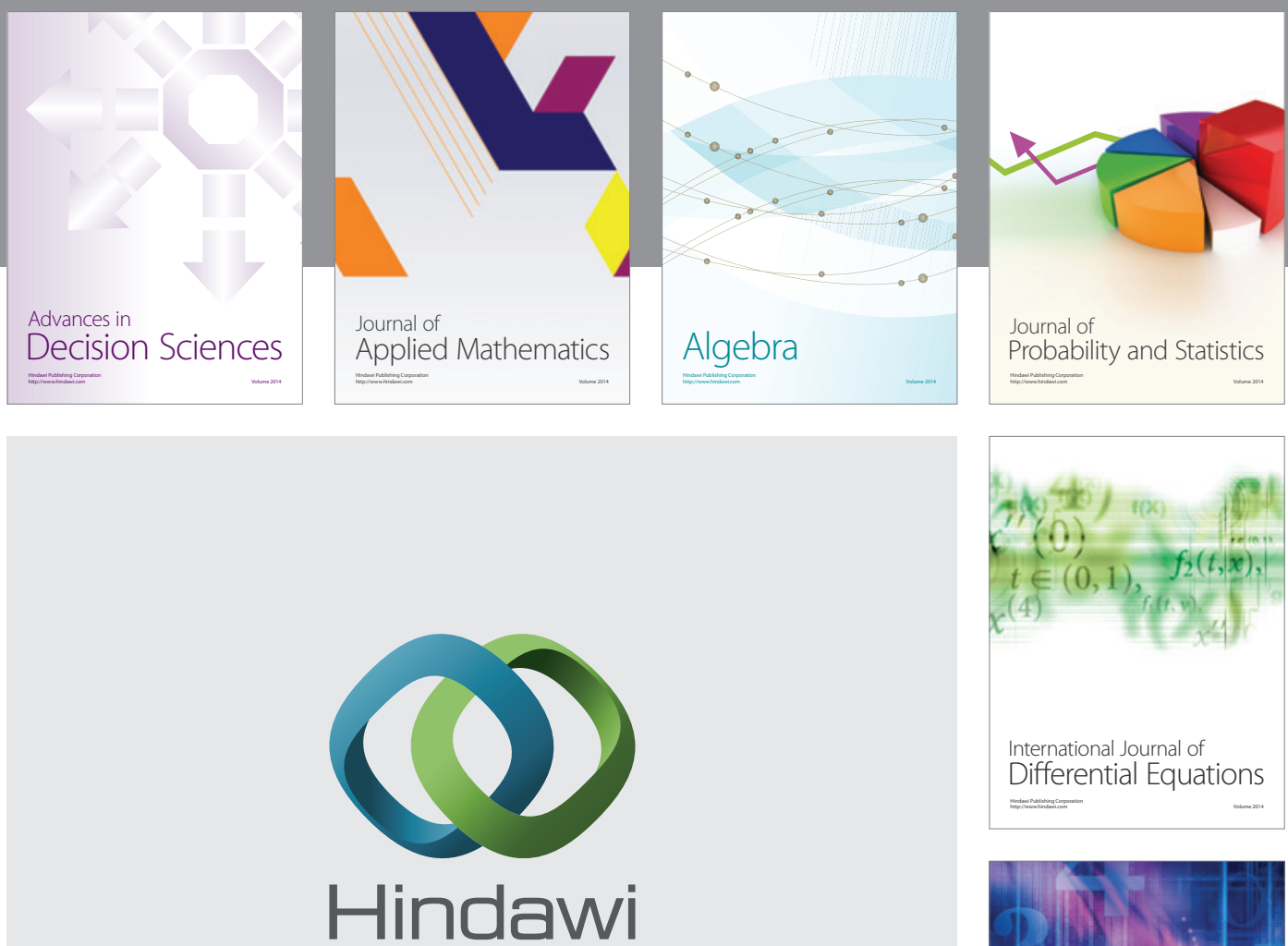

Submit your manuscripts at http://www.hindawi.com
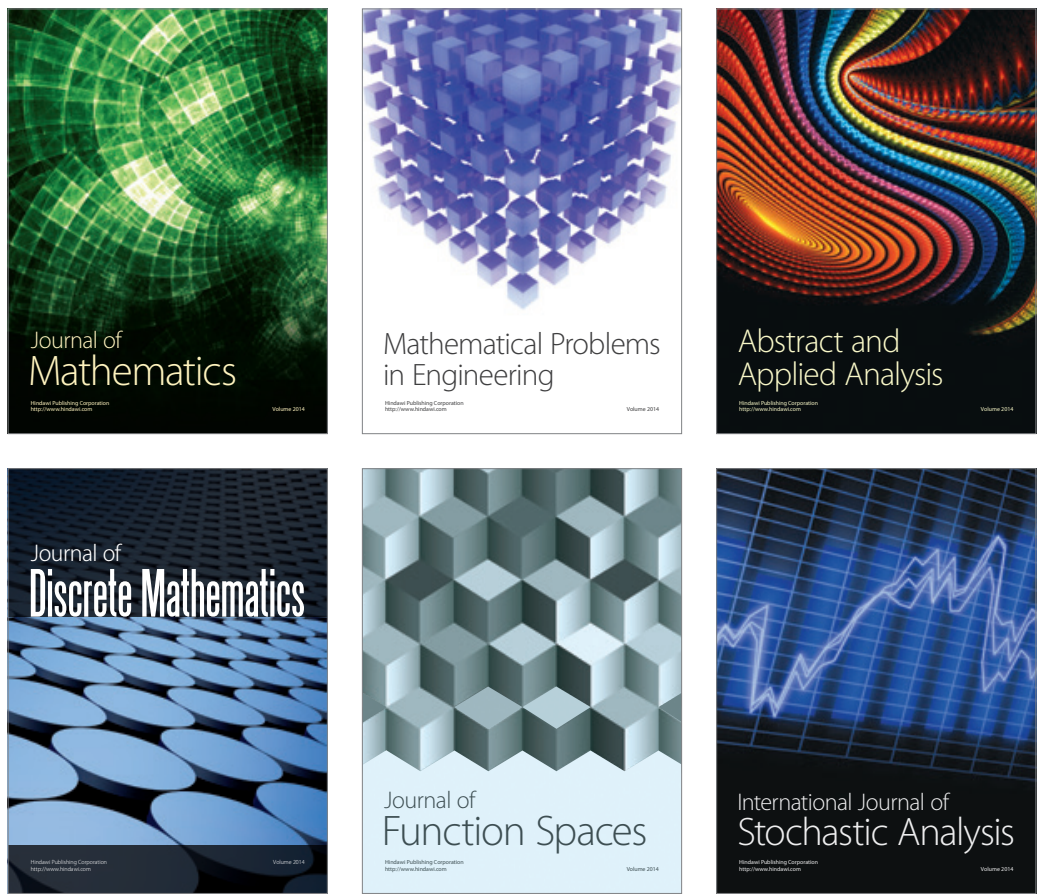

Journal of

Function Spaces

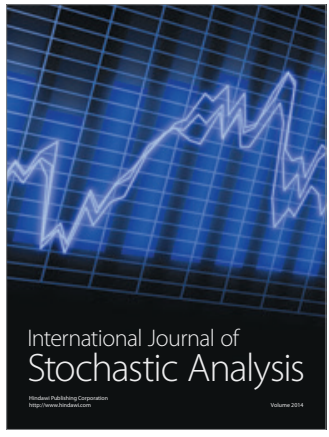

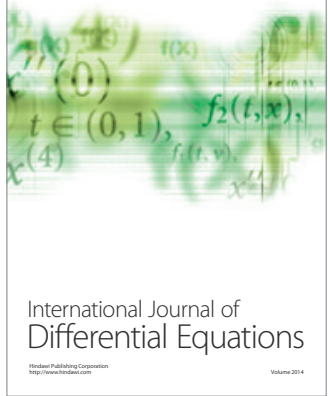
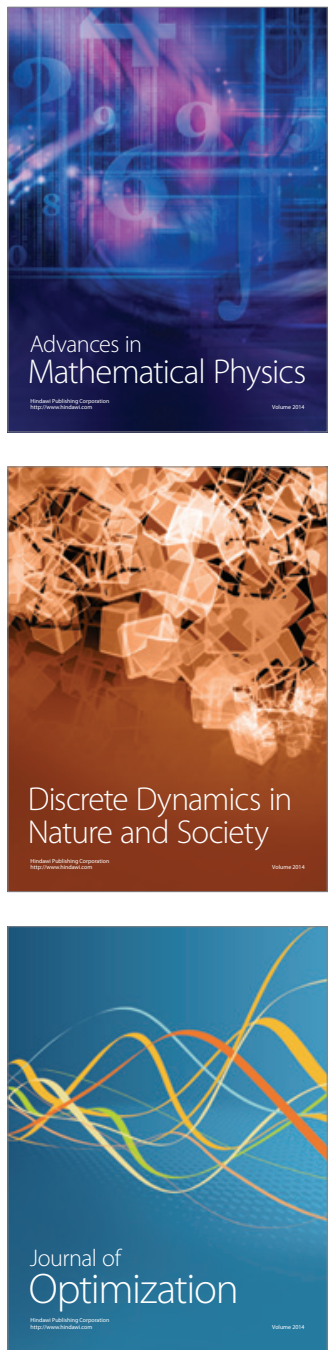